\title{
Tendinopatía del tendón flexor digital superficial y desmopatía del ligamento suspensorio en caballos: fisiopatología y terapias regenerativas
}

\author{
Superficial digital flexor tendon tendinopathy and suspensory ligament desmopathy \\ in horses: pathophysiology and regenerative therapies
}

\author{
JU Carmona ${ }^{a^{*}}$, C López ${ }^{a, b}$ \\ ${ }^{a}$ Grupo de Investigación Terapia Regenerativa, Departamento de Salud Animal, Universidad de Caldas, Manizales, Colombia. \\ bBecaria COLCIENCIAS “Generación Bicentenario", Programa de Doctorado en Ciencias Biomédicas, Universidad de Caldas, \\ Manizales, Colombia.
}

\begin{abstract}
SUMMARY
Tendon and ligament injuries are frequent in horses. The superficial digital flexor tendon (SDFT) and the suspensory ligament (SL) are the most frequently affected structures. The clinical form of these pathologies is generally chronic and degenerative. The treatments commonly used do not result in a definitive cure of the problem and most of the patients relapse or do not recover their initial athletic capacity. Advances in molecular pathophysiology of tendonitis and desmitis in man, horses and other animals have shown the presence of catabolic cytokines, which are possibly responsible for the general dysfunction observed in these pathologies. The current objectives of the treatments of these problems are the regeneration and not the repair (scar formation) of the injured tissue. In the horse, novel experimental and clinical treatments have been described. These treatments include the injection of bone marrow aspirates, mesenchymal stem cells, recombinant growth factors such as insulin-like and platelet rich plasma (also termed autologous platelet concentrates), amongst others. The observed results have been promising when these novel therapies were used. However, just as with other treatments, further research is needed to demonstrate that its clinical use can be effective and safe in horses.
\end{abstract}

Palabras clave: equino, tendinopatía, desmopatía, tejido conectivo, medicina regenerativa.

Key words: equine, tendinopathy, desmopathy, connective tissue, regenerative medicine.

\section{INTRODUCCIÓN}

Las enfermedades más comunes en el caballo son aquellas que afectan su aparato musculoesquelético. Estudios realizados en caballos de carreras sugieren que estas lesiones producen importantes pérdidas económicas (Halper y col 2006) y que representan cerca del $82 \%$ de los problemas de pérdida de rendimiento manifestado clínicamente como cojera; entre el 46 y el 53\% son lesiones de tendones y ligamentos (Thorpe y col 2010). Estos sanan muy lentamente y el tejido reparado no tiene las mismas características de elasticidad y fuerza que el tejido original (Dahlgren 2007). Uno de los principales dilemas relacionados con las tendinopatías y desmopatías del caballo es la elección del tratamiento (Smith y Schramme 2003, Dowling y Dart 2005). Muchos veterinarios toman decisiones terapéuticas basados en sus experiencias clínicas (Paavola y col 2002) y pocas en el conocimiento actual de la fisiopatología de tendones y ligamentos lesionados (Smith y Schramme 2003).

El tratamiento médico convencional de estas patologías en el caballo incluye la utilización de antiinflamatorios no esteroideos (AINEs), glicosaminoglicanos polisulfatados

\footnotetext{
Aceptado: 28.04.2011.

* Calle 65 N $^{\circ}$ 26-10, Manizales, Caldas, Colombia; carmona@ucaldas. edu.co
}

(PSGGs), inyección de corticosteroides (CS), ácido hialurónico (HA) y de fumarato de $\beta$-aminopropionitrilo (BAPN) (Ross y Dyson 2002, Smith y Schramme 2003, Dyson 2004, Gaujoux-Viala y col 2009).

En los casos de tendinopatía y desmopatía agudas se emplean fármacos combinados con reposo, hidroterapia y vendajes a presión. En la fase subaguda de la tendinopatía del tendón flexor digital superficial (SDFT) se utilizan HA o BAPN, combinados con monitorización ecográfica de la lesión y un programa de ejercicio controlado (Dowling y col 2000, Smith y Schramme 2003, Dyson 2004). Algunos clínicos también usan en esta fase las ondas de choque, rayos infrarrojos y láser frío, entre otros. La utilización de CS como tratamiento de estas lesiones es un tema controversial. Existen datos que desaconsejan su empleo, ya que aumentan el riesgo de roturas del tendón (Paavola y col 2002, Chang y col 2010). Sin embargo, muchos veterinarios y médicos humanos persisten con su uso (Dowling y col 2000, Smith y Schramme 2003, GaujouxViala y col 2009, Peters-Veluthamaningal y col 2009).

En casos crónicos, cuando estos tratamientos fallan, el clínico puede recurrir a la cirugía (Dowling y col 2000, Smith y Schramme 2003, Kaneps 2007) o a otros procedimientos poco convencionales como la aplicación de puntos de fuego (menos usado y no justificado), en el caso de las tendinopatías. Los procedimientos quirúrgicos más usados en la tendinopatía del tendón 
flexor digital superficial (SDFT) son la estimulación de la revascularización de las lesiones crónicas y la desmotomía del ligamento accesorio (AL) del tendón flexor digital superficial (AL-SDFT) (Kaneps 2007). Aunque ambas cirugías fueron ampliamente utilizadas, actualmente su uso no está justificado (Dowling y col 2000, Smith y Schramme 2003). Las lesiones del ligamento suspensor (SL), especialmente de los miembros posteriores y que son refractarias al tratamiento médico, han sido manejadas mediante fasciotomía plantar profunda e incluso neurectomía del nervio tibial o de la rama profunda lateral del nervio plantar (Dyson y col 1995, Dyson y Genovese 2003, Kaneps 2007). Recientemente, se ha demostrado que las neurectomías producen atrofia por denervación del SL y podrían predisponer a una rotura catastrófica de esta estructura anatómica (Pauwels y col 2009).

Los mecanismos de reparación natural no permiten que los tendones y ligamentos lesionados se recuperen completamente. Esto hace que los caballos afectados con estos problemas tengan una predisposición de recaída aproximadamente del $80 \%$ (Halper y col 2006), a pesar de emplear cualquier tipo de tratamientos convencionales (clásicos) o terapia física. Esta situación no es diferente a lo que sucede en seres humanos afectados por tendinopatías o desmopatías, especialmente crónicas (Paavola y col 2002).

Durante los últimos años se ha incrementado el conocimiento de la biología del tejido conectivo y se han podido esclarecer a nivel molecular varios procesos bioquímicos relacionados con la fisiopatología de estas lesiones (Goodship y col 1994, Kobayashi y col 1999, Abramowitch y col 2003, Woo y col $2008^{a}$,b Souza y col 2010). Esto ha permitido esclarecer, en parte, la causa de los fracasos obtenidos con la terapéutica convencional y ha abierto una nueva era en el tratamiento de estas lesiones, la era de la terapia regenerativa.

En este trabajo se presenta una revisión de los principales tratamientos regenerativos (experimentales y clínicos) que se han propuesto como terapia para tendinopatías y desmopatías del caballo, y para ello se describe previamente la morfofisiología de los tendones y ligamentos de este animal.

\section{MORFOFISIOLOGÍA DE LOS TENDONES Y LIGAMENTOS DEL CABALLO}

La anatomía de los tendones y ligamentos varía entre miembros anteriores y posteriores y entre caballos. Sin embargo, es muy similar en la región digital de ambos miembros (figura 1). En cada extremidad se pueden identificar tres partes anatómicas principales: el SDFT y su AL-SDFT, el tendón flexor digital profundo (DDFT) y su ligamento accesorio (AL-DDFT) y el aparato suspensorio (SA), compuesto por el tercer músculo interóseo (SL), el escudo proximal, los ligamentos sesamoideos distales (DSLs) y la brida extensora (Denoix 1994).

Los tendones y ligamentos son estructuras muy parecidas desde el punto de vista ultraestructural. Los tendones son continuación de los músculos y están formados por una trama de colágeno que da soporte a las

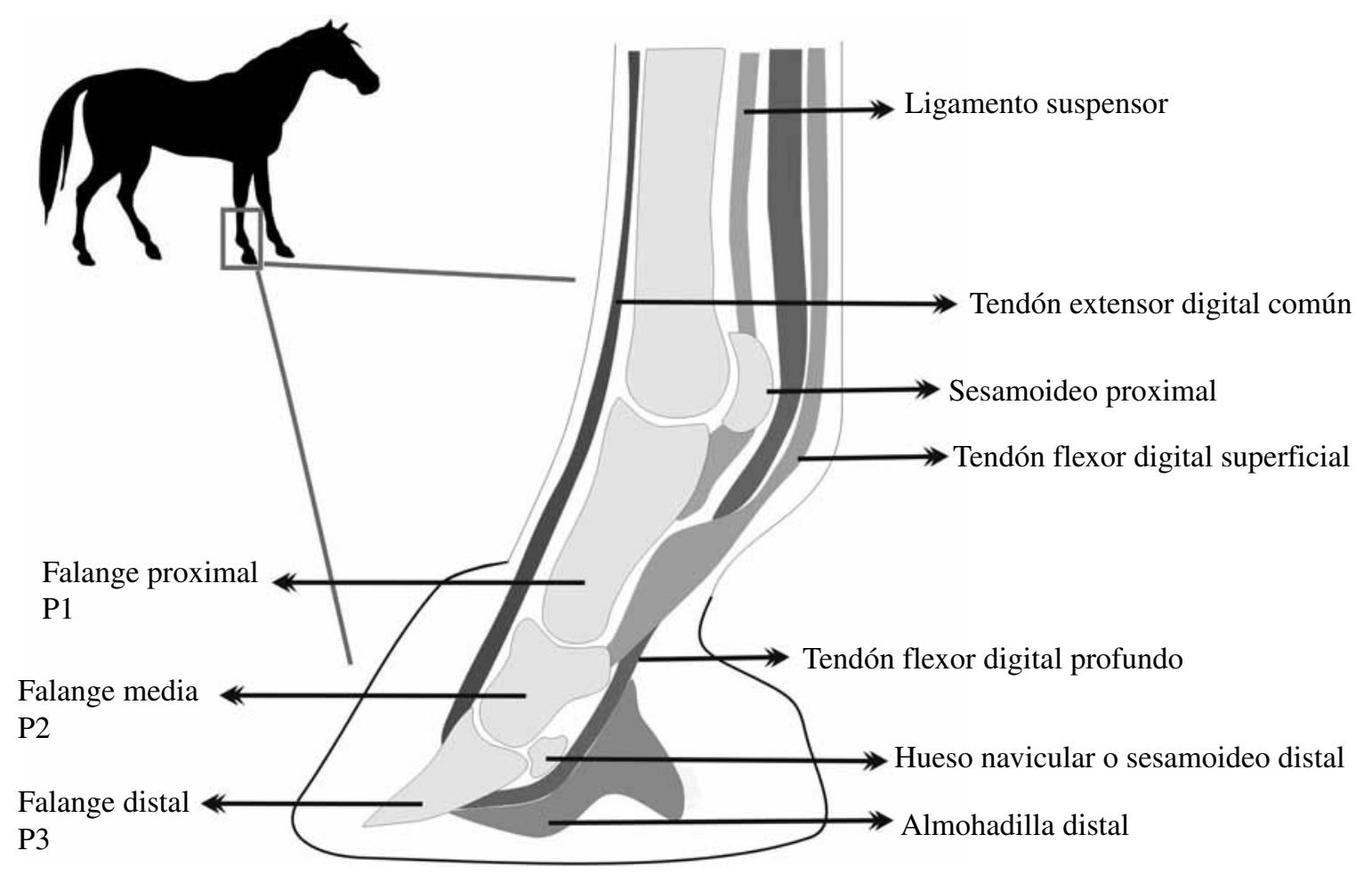

Figura 1. Anatomía básica de un dígito en el caballo. Basic anatomy of a digit in the horse. 
fibras musculares contráctiles. Aparecen como bandas o cordones blancos de tejido conectivo denso que conectan el vientre muscular a un elemento esquelético distal. El corte transversal de una superficie tendinosa revela la presencia de septos interfasciculares. Los fascículos varían en tamaño y forma y funcionan de manera independiente. Están formados por fibrillas y éstas a su vez por subfibrillas. Las subfibrillas están compuestas por microfibrillas de tropocolágeno (figura 2) (Kastelic y col 1978, Thorpe y col 2010).

Las células que forman los tendones son fibroblastos especializados, los tenocitos. Están representados por tres poblaciones celulares específicas que cumplen diferentes funciones bioquímicas dentro del tendón, pero principalmente generan matriz extracelular (Goodship y col 1994, Dowling y col 2000, Smith y Schramme 2003, Dahlgren 2007). Los tendones también son infiltrados por pequeños vasos sanguíneos que recorren los espacios interfasciculares de las fibras de colágeno. La normal vascularización del tendón favorece el transporte de nutrientes, enzimas proteolíticas, mediadores de la inflamación, entre otros (Bosch y col 2010).

La matriz extracelular (ECM) del tendón o ligamento está conformada por diferentes tipos de macromoléculas: colágeno, elastina, proteoglicanos, glicoproteínas y agua. El colágeno y la elastina forman el componente fibroso que da fuerza tensil al tendón y al ligamento (Dahlgren 2007). La molécula de colágeno (tropocolágeno) está formada por tres cadenas de polipéptidos enrolladas hacia la derecha en forma de superhélice (figura 2). En los tendones y ligamentos existe una gran proporción de colágeno tipo I (Col-I) (95\%) (Dahlgren 2007), aunque también se encuentran pequeñas proporciones de Col-II, III, IV, V y VI. El Col-II se expresa particularmente en las zonas donde el tendón sufre fuerzas de compresión, tales como la región metacarpiana o a su paso entre los huesos sesamoideos. Por otro lado, el Col-III predomina después de las lesiones y traumatismos, en procesos reparativos tempranos y forma microfibrillas de pequeño diámetro (Goodship y col 1994, Dowling y col 2000, Smith y Schramme 2003).

El colágeno se organiza a manera de fibrillas en paquetes de $67 \mathrm{~nm}$. La organización depende de la colocación de cada haz en sentido hidrofílico e hidrofóbico y de las uniones entre cada molécula. Los proteoglicanos y las glicoproteínas influyen sobre la matriz de colágeno y sobre la agregación de las fibrillas de colágeno (Goodship y col 1994, Dahlgren 2007). En estado de relajación, las fibrillas de colágeno presentan forma de espiral (resorte o crimp). El crimp imparte elasticidad al tendón en las fases tempranas de carga $\left(19-20^{\circ}\right.$ y $\left.17-19 \mu \mathrm{m}\right)$ y esta disminuye con la maduración del tendón o con el ejercicio (12-17 ${ }^{\circ}$ y 11-15 $\mu \mathrm{m}$ ) (Dowling y col 2000). A los dos años de edad el tendón es morfológicamente maduro y presenta características mecánicas de mayor rigidez (disminución en el ángulo de elasticidad, disminución en el tamaño de los fascículos) (Patterson-Kane y col 1997). Esta disminución en la elasticidad y aumento de la rigidez del tendón podrían favorecer la presentación de tendinopatías en el caballo. Por otra parte, el diámetro (forma) y el número de las fibrillas disminuye con la edad, lo que predispone a los caballos viejos a sufrir lesiones tendinosas (Birch y col 1999, Edwards y col 2005).

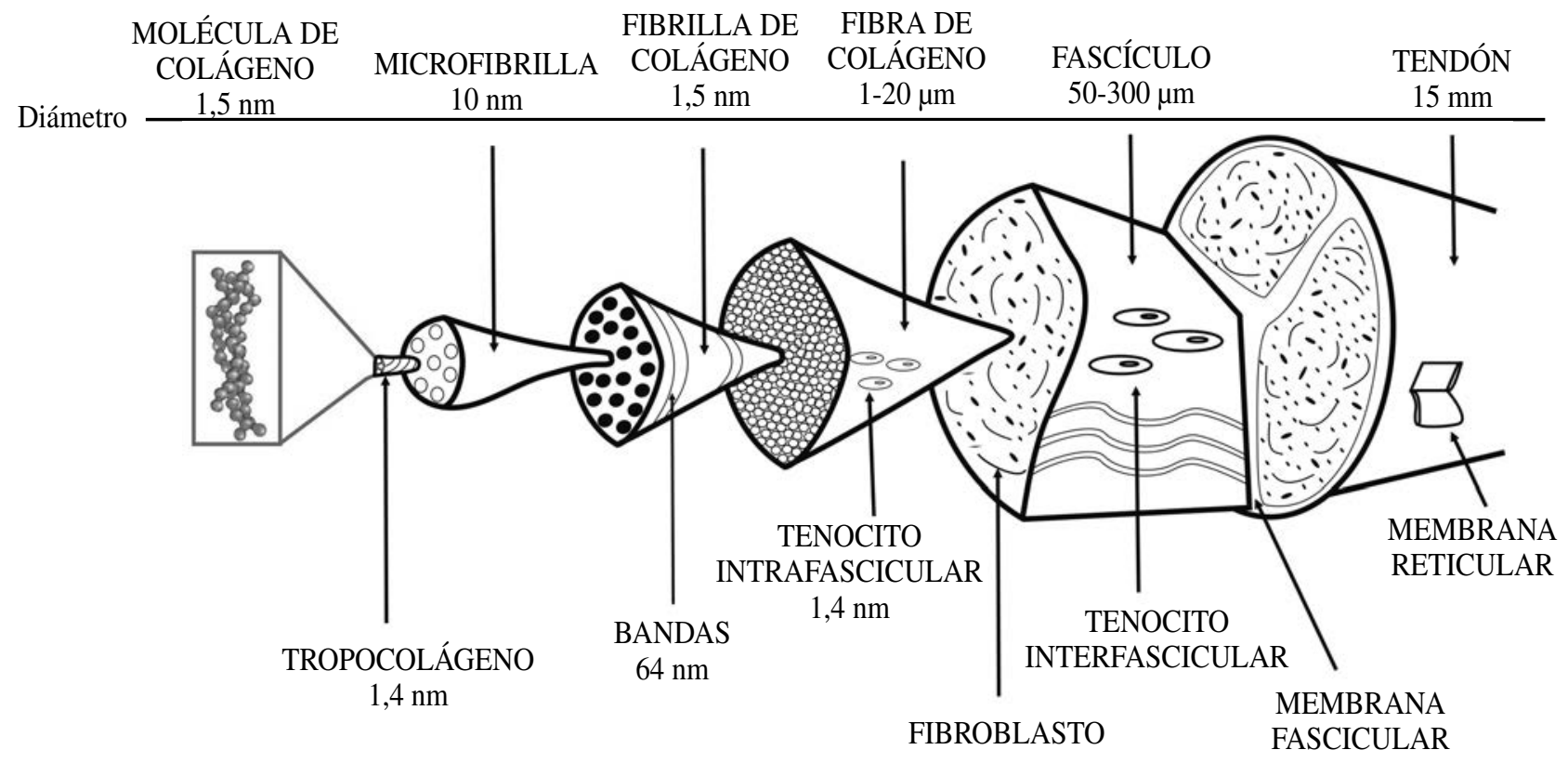

Figura 2. Organización microestructural y funcional del tendón (modificado de Kastelic y col 1978 y Thorpe y col 2010). Microstructural and functional organization of the tendon (modified from Kastelic et al 1978 and, Thorpe et al 2010). 


\section{BIOMECÁNICA}

Los tendones y ligamentos del caballo son estructuras muy fuertes que soportan altas cargas y tensiones durante la estación y el movimiento (Denoix 1994). El aparato elástico que forman se encarga principalmente de dar soporte al menudillo, prevenir la hiperextensión del carpo, amortiguar la energía del impacto y sostener completamente el peso corporal durante la propulsión (Dowling y col 2000).

Los tendones y ligamentos de caballos jóvenes se adaptan a las diferentes cargas producidas en su desarrollo (Smith y col 1999). Sin embargo, a medida que estos alcanzan la madurez esquelética (5 años) (Dahlgren y col 2002), muchos de ellos son propensos a acumular microlesiones a causa de la fatiga constante producida en su actividad atlética (Pool y Meagher 1990, PattersonKane y col 1998). Smith y col (2002) estudiaron la distribución de la proteína oligomérica de la matriz del cartílago (COMP) en tendones de caballo. Esta proteína se encuentra en gran cantidad en los tendones de animales en desarrollo, por lo que se cree que es importante en la formación de la ECM del tendón en los primeros estadios de vida. La COMP disminuye a medida que el animal envejece y es posible que este fenómeno lo haga más susceptible a padecer lesiones tendinosas. En caballos se encontró que su cantidad difiere entre poblaciones y quizás por esto se podría explicar el amplio rango de variación en las propiedades mecánicas de los tendones entre individuos y razas de equinos (Crevier-Denoix y col 1997, Smith y col 2002).

Durante la locomoción, los tendones flexores y el SL actúan como un mecanismo elástico que disipa energía e incrementa el rendimiento durante la marcha (Denoix 1994) y actúan como resortes para proteger las fibras musculares durante movimientos fuertes, súbitos o inesperados (figura 3) (Goodship y col 1994).

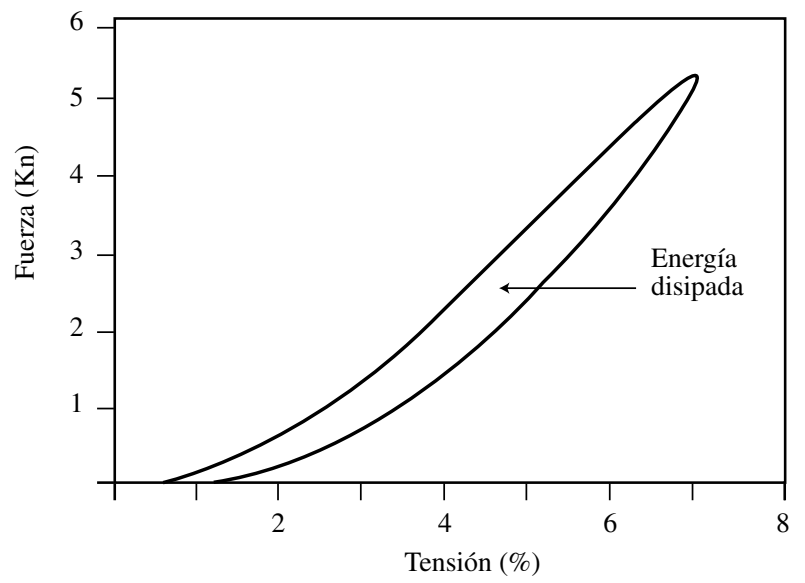

Figura 3. Ciclo de carga del tendón flexor digital superficial en un caballo (adaptado de Goodship y col 1994).

Charging cycle of the superficial digital flexor tendon in a horse (adapted from Goodship et al 1994).
Los tendones son altamente ténsiles, fuertes, flexibles y elásticos y al igual que los ligamentos, exhiben características de deformación no lineares frente a la carga. Esto se traduce a que pequeños incrementos en la carga pueden producir gran extensión del tendón, hasta un límite o punto crítico. Después de cada incremento en la carga, se genera un cambio similar en la extensión. Si la carga cesa en ese punto, la estructura vuelve a su estado normal y elimina la energía en forma de calor (figura 4). Si la carga continúa después del punto crítico, se produce el fenómeno llamado deformación plástica y finalmente el tendón o ligamento fallan y se rompen (Goodship y col 1994, Dowling y col 2000, Smith y Schramme 2003, Smith y col 2003).

El componente no linear de la fuerza elástica de los tendones y ligamentos se debe a las características microestructurales de las fibrillas de colágeno (Goodship y col 1994). El valor de la resistencia elástica del tendón es muy estrecho. La rotura del SDFT ocurre cuando se exceden las cargas de tolerancia fisiológica por encima de un $12-20 \%$. Por esta razón, el nivel máximo de rendimiento en un equino atleta puede estar cercano a ese punto crítico de rotura (figura 4) (Goodship y col 1994). En el SDFT se presenta inicialmente daño de su región central. Este fenómeno se debe principalmente a procesos degenerativos que intervienen con la calidad de la formación de las espirales del colágeno y es común en caballos viejos (Crevier-Denoix y col 1997).

Estudios ex vivo han mostrado que el cuerpo del SL puede romperse cuando se excede su carga entre 10-12\% (Goodship y col 1994). En situaciones in vivo, el aparato suspensor de los miembros anteriores es normalmente el más afectado por roturas catastróficas (Thorpe y col 2010). Éstas se pueden localizar en el cuerpo del SL o cerca de la región de los huesos sesamoideos proximales, especialmente al lado del cóndilo lateral del tercer hueso metacarpiano.

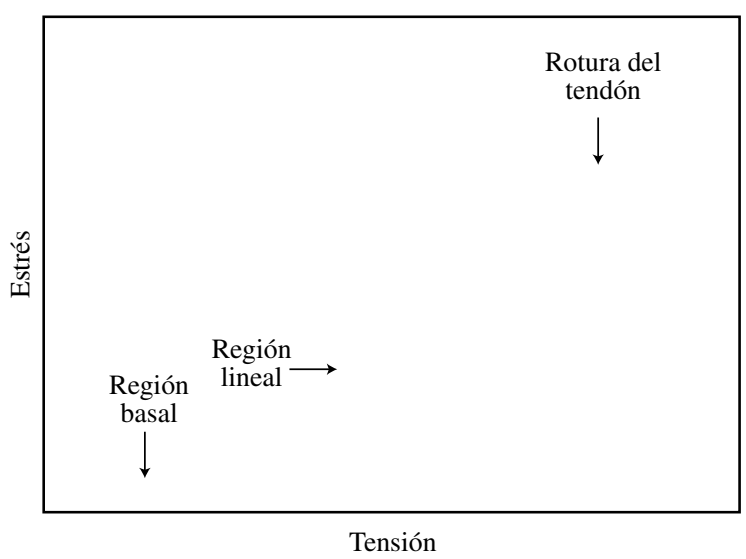

Figura 4. Curva de carga y rotura de un tendón equino (adaptado de Goodship y col 1994).

Charging curve and rupture of an equine tendon (adapted from Goodship and col 1994). 


\section{BIOPATOLOGÍA DE LAS LESIONES DE TENDONES Y LIGAMENTOS}

Las lesiones del SDFT representan más del 30\% de las lesiones de equinos atletas en Europa (Pool y Meagher 1990, Wong y col 2003). Las afecciones pueden ir desde roturas parciales menores a rotura bilateral completa de tendones. Los SDFTs de los miembros anteriores son generalmente más afectados (Pool y Meagher 1990, Dowling y col 2000, Smith y Schramme 2003, Wong y col 2003). Lesiones similares también pueden ocurrir en los miembros posteriores, aunque en este caso son más frecuentes las lesiones del DDFT y SL (Goodship y col 1994). Por su parte, las lesiones del SL pueden representar hasta el 3,6\% en caballos purasangre inglés (Dyson 2004).

Las desmopatías de los SLs de los miembros anteriores son más frecuentes en caballos de carrera, mientras que las desmitis del LS de los miembros posteriores son más frecuentes en trotadores. La desmitis bilateral del SL de los miembros anteriores puede llegar a presentarse en el $31 \%$ de los caballos afectados. Este mismo trastorno en los miembros posteriores puede representar un $20 \%$ de los caballos afectados (Dyson 2004).

El daño preferencial de un tendón o un ligamento dependerá en gran medida de la carga funcional de estas estructuras en diferentes pasos o aires y en asociación con diferentes demandas locomotoras (Denoix 1994). Las fuerzas impuestas sobre los tendones y ligamentos son modificadas por el tipo de actividad, gradientes del terreno, superficie, tipo de herraje aplicado al casco y la conformación del caballo (Goodship y col 1994, Anderson y col 2004). Además, la edad, el nivel de entrenamiento y la historia de ejercicio están implicados en la génesis de la lesión (Goodship y col 1994, Dowling y col 2000, Smith y Schramme 2003).

Las tendinopatías del SDFT pueden clasificarse como agudas (tendinitis) o crónicas (tendinosis). Las lesiones del SDFT pueden comprender desde una degeneración subclínica de la región central del tendón, hasta rotura completa del mismo (Pool y Meagher 1990, Kobayashi y col 1999). La lesión puede surgir a partir de una carga mecánica sobreaguda, como causa de un movimiento no coordinado (factores extrínsecos) o por estrés acumulado a partir de una lesión subclínica repetida (factores intrínsecos) (Smith y Schramme 2003). La lesión degenerativa (tendinosis o tendinopatía) del SDFT guarda gran semejanza con la tendinopatía del tendón de Aquiles en seres humanos (Dahlgren y col 2002, Paavola y col 2002, Birch y col 2008). En ambas patologías se aprecia deterioro generalizado de la ECM, con disminución significativa del Col-I, ausencia de reacción (infiltración) inflamatoria del tendón y vascularización disminuida.

Las desmopatías del SL también pueden clasificarse de igual manera que las tendinopatías. La gravedad de las lesiones del SL dependerá de su magnitud, región afectada y grupo de miembros comprometidos. Las desmopatías proximales del SL (PDSL) de los miembros anteriores son menos drásticas que las de los miembros posteriores (Denoix 1994). En general, los caballos afectados por PDSL de los miembros anteriores pueden retornar a su nivel de entrenamiento normal, después de reposo y un programa de ejercicio controlado durante tres meses. Los caballos que padecen este problema en los miembros posteriores tienen muy mal pronóstico, continúan con diferentes grados de cojera y casi nunca regresan a su nivel de entrenamiento normal (Dyson y col 1995, Ross y Dyson 2002).

Las desmopatías de las ramas del SL (BDSL) de los miembros anteriores son más frecuentes que en los miembros posteriores y están asociadas con un proceso crónico. Algunas lesiones de este tipo sanan muy lentamente (algunas pueden llegar hasta 18 meses) y muchos caballos no retornan a su nivel de trabajo normal, hasta 9 meses de haberse producido la lesión (Dyson y col 1995, Ross y Dyson 2002). Las desmopatías de las BDSL de los miembros posteriores se presentan más a menudo en caballos ambladores (ej.: paso peruano y paso colombiano), de doma clásica y en animales rectos de corvejones o largos de cuartillas (pandos).

Los tendones y ligamentos, después de lesionados, cicatrizan lentamente y nunca vuelven a recuperar sus características biomecánicas originales (Goodship y col 1994, Crevier-Denoix y col 1997). Cuando un caballo ha sufrido un episodio de tendinitis o desmitis es muy probable que se vuelva a repetir la lesión, en un grado semejante a la anterior o incluso con mayores consecuencias (Dowling y col 2000, Smith y Schramme 2003). Se han propuesto diferentes teorías (mecanismos) para tratar de explicar la causa de la lesión central degenerativa del SDFT equino. Estos mecanismos se pueden dividir en físicos y bioquímicos. Las causas bioquímicas pueden incluir mecanismos por hipoxia-isquemia, reperfusión, entre otros. Las causas físicas incluyen sobrecarga e hipertermia (Goodship y col 1994, Dowling y col 2000, Smith y Schramme 2003, Carmona y col 2009).

Es importante considerar, independientemente de la causa inicial, que se producen cambios bioquímicos (ej.: expresión de citocinas catabólicas y activación exagerada de las metaloproteinasas de matriz (MMPs)) y celulares (ej.: apoptosis de tenocitos y proliferación de miofibroblastos, entre otros) (Goodship y col 1994, Paavola y col 2002, Carmona y col 2009, Thorpe y col 2010) que producen impactos estructurales y funcionales negativos sobre el tendón, que con el tiempo terminan en una lesión clínica (Dowling y col 2000, Smith y Schramme 2003). De esta manera, se sabe que los tendones rotos (o próximos a rotura) presentan aumento de la expresión génica y producción exagerada de MMP1 (colagenasa), mientras que la expresión génica de la MMP-3 (estromelisina) se encuentra disminuida (Thorpe y col 2010). 
Después de la lesión, el proceso de reparación del tendón o ligamento sigue los mismos patrones básicos que se observan en la mayoría de los tejidos corporales. La rotura fibrilar inicial es seguida por hemorragia y formación de un hematoma con la consecuente formación de una malla de fibrina, acompañado de edema e inflamación. Luego, llegan los macrófagos que eliminan los residuos del proceso inflamatorio y de manera superpuesta los fibroblastos comienzan a formar una cicatriz. En el nuevo tejido de cicatrización se presenta una deposición inicial desorganizada de colágeno inmaduro $($ Col-III). Posteriormente, se presenta un proceso de maduración con incremento de fibrillas de Col-I, que poseen mayor diámetro y son organizadas en distribución paralela, más parecida a la forma tendinosa original. Este proceso puede durar de semanas a meses (Goodship y col 1994, Dowling y col 2000, Smith y Schramme 2003, Dahlgren 2007).

\section{TERAPIA REGENERATIVA}

La medicina regenerativa se centra en estrategias terapéuticas que permitan reemplazar o restaurar tejidos dañados o contrarrestar el envejecimiento de las células en el cuerpo y así mejorar su función (Gurtner y col 2007). La regeneración implica la reposición de células originales (del tejido lesionado), después de una sección o una lesión, seguida por la generación de células progenitoras de regeneración y morfogénesis (Brockes y Kumar 2008). Su principal objetivo es obtener la regeneración de los tejidos y evitar la reparación (cicatrización) de los mismos.

Diferentes revisiones sobre la fisiopatología de las lesiones de tendones y ligamentos han hecho énfasis sobre la influencia de los factores físicos (ej: estrés mecánico e hipertermia) en el deterioro de estas estructuras (Goodship y col 1994, Henninger 1994, Smith y Schramme 2003, Smith y col 2003, Carmona y col 2009). Sin embargo, actualmente es necesario incluir algunos factores bioquímicos, que anteriormente habían sido tímidamente considerados dentro de este mecanismo patológico.

Recientemente se describió la presencia de citocinas catabólicas (factor de necrosis tumoral alfa (TNF- $\alpha$ ) e interleucina 1 alfa (IL-1 $\alpha$ ) y beta (IL-1 $\beta$ ) en biopsias de tendones de equinos con tendinopatía crónica del SDFT (Hosaka y col 2002). Tsuzaki y col (2003) demostraron in vitro en tenocitos humanos que el desafío exógeno con IL-1 estimula la expresión de IL-1 propia y, a su vez, IL-6, ciclo-oxigenasa 2 (COX-2), MMPs y todas las consecuencias catabólicas que conlleva la expresión de éstas moléculas. Además, se ha encontrado que algunos factores de crecimiento (GFs), como el factor de crecimiento transformador beta (TGF- $\beta$ ) disminuyen sus concentraciones en la ECM del tendón, a medida que el animal envejece. Se sabe que este factor es crucial en la síntesis de colágeno (Smith y Schramme 2003). Sin embargo, parece que tuviera una función dual en la fisiopatología de las tendinopatías del caballo, ya que su expresión exagerada puede inducir fibrosis y alterar la producción de colágenos juveniles o embrionarios (tipos XII y XIV) en el tendón (Arai y col 2002).

Es probable que el mecanismo molecular de las tendinopatías y desmopatías del caballo pueda tener similitud con la fisiopatología de la osteoartritis (OA) (Carmona y Prades 2009). La OA es una enfermedad crónica en la que se produce una respuesta reparativa insuficiente del cartílago articular (Carmona y col 2009) debido a un desequilibrio entre los péptidos que inducen la síntesis y degradación de la ECM del cartílago articular.

El cartílago es una estructura de tejido conectivo modificado, compuesto por células especializadas (condrocitos) embebidas en una ECM rica en Col-II. La estructura general del cartílago, tendones y ligamentos es muy parecida (Carmona y Prades 2009). No obstante, en la ECM de estas dos últimas estructuras predomina el Col-I (Goodship y col 1994). Se sabe que en esta clase de ambiente tisular las células interactúan entre sí por medio de numerosos mensajeros celulares (citocinas), GFs y diversas enzimas proteolíticas. Esta interacción es muy importante para mantener la salud del tejido, puesto que se asegura un equilibrio entre la proliferación de las células residentes, la síntesis y degradación de los diferentes componentes de la ECM (Carmona y col 2009, Carmona y Prades 2009).

A la luz del concepto teórico anterior, se podría tratar de explicar la causa del fracaso de los tratamientos convencionales de las tendinopatías y desmopatías en el caballo, especialmente en sus fases crónicas. En general, la farmacoterapia con NSAIDs y CS sólo puede actuar de manera sintomática e incluso estas sustancias podrían agravar la lesión, al inhibir la síntesis de moléculas (ej., prostaglandinas) o la expresión de genes clave (ej., genes de las ciclooxigenasas) en los procesos de resolución de la inflamación, reparación o regeneración del tendón o ligamento, tal como se ha observado en la OA. También sería lógico pensar que los tratamientos con HA y PSGGs podrían ser más racionales en el manejo de estas patologías, ya que ambas sustancias son componentes naturales de la ECM. Sin embargo, se ha demostrado extensamente que estos fármacos no representan una ventaja terapéutica frente al reposo o a un programa de ejercicio controlado en caballos con tendinopatías o desmopatías (Dowling y col 2000, Smith y Schramme 2003, Dyson 2004).

Actualmente, se han desplazado las sustancias con un efecto sintomático y se ha iniciado la búsqueda de fármacos o compuestos biológicos con efectos regenerativos: "terapia regenerativa". Durante los últimos años se han producido avances significativos en este campo en el caballo y otras especies animales. En equinos se han realizado diferentes investigaciones in vitro (Nixon y col 1999, Dahlgren y col 2001) e in vivo (Herthel 2001, Dahlgren y col 2002) sobre algunos enfoques de 
terapia regenerativa en lesiones del SL y SDFT. También se ha documentado una investigación clínica sobre el uso de concentrados de plaquetas como tratamiento de desmopatías crónicas del SL (Carmona y col 2009) y del cuerpo del SL (Waselau y col 2008); la inyección intralesional de células mesenquimales autólogas como tratamiento de un caso de tendinosis del SDFT (Smith y col 2003) y un estudio experimental sobre el efecto de la hormona del crecimiento recombinante equina (re-GH) (Dowling y col 2002). En otras especies animales (estudios in vivo e in vitro) se ha investigado el efecto individual y la combinación de varios GFs en el metabolismo de tendones y ligamentos (cuadro 1) (Zhang y col 2003).

\section{FACTORES DE CRECIMIENTO}

Los factores de crecimiento (GFs) son péptidos producidos por células, que pueden actuar de manera autocrina, paracrina y endocrina. Estas moléculas son mal denominadas GFs, ya que cuando algunas de ellas fueron aisladas por primera vez se observó que producían proliferación celular in vitro. Sin embargo, actualmente se reconoce que no sólo estimulan la proliferación celular, sino que también son clave en diversos procesos, como la cicatrización y angiogénesis. Por esta razón se deberían denominar como péptidos polifuncionales.

Los GFs han ganado gran interés en el campo de la terapia regenerativa de numerosas patologías del hombre y los animales, tales como la OA (Platt y col 1998, Carmona y Prades 2009); la cicatrización patológica de la piel y otros tejidos (Nam y col 2010); las tendinopatías y desmopatías (Zhang y col 2003), entre otras. Los GFs pueden antagonizar los efectos catabólicos y proinflamatorios de la IL-1 o del TNF $\alpha$ (Van Miert 2002).
Estas citocinas están implicadas en la génesis o en la fisiopatología de varias enfermedades (ej. OA) (Carmona y Prades 2009). Además, como se mencionó anteriormente, posiblemente podrían jugar un papel importante en la biología de las lesiones de los tejidos blandos del aparato locomotor (Hosaka y col 2002).

Diferentes investigaciones, in vitro e in vivo (cuadro 1) en humanos y otras especies animales, han demostrado efectos positivos de varios GFs sobre el metabolismo de tendones y ligamentos (Murray y col 2003). En general, el factor de crecimiento fibroblástico (FGF), factor de crecimiento vasculoendotelial (VEGF) y el factor de crecimiento transformador beta (TGF- $\beta$ ) producen angiogénesis en el sitio de la lesión (Carter y col 2003, Murray y col 2003, Carmona y col 2009). El factor de crecimiento derivado de las plaquetas (PDGF), IGF-I, FGF y el factor de crecimiento epidérmico (EGF) estimulan migración y mitosis de los tenocitos (Murray y col 2003, Tang y col 2003, Wong y col 2003, Zhang y col 2003). El TGF- $\beta$, IGF-I y FGF estimulan la síntesis de ECM (Spindler y col 2003).

Factor de crecimiento insulínico tipo-I. El IGF-I pertenece a la familia de péptidos relacionados con la insulina, esenciales para un normal desarrollo y crecimiento (Verwilghen y col 2009). Este factor de crecimiento, es regulado por el eje de la hormona del crecimiento y producido principalmente por el hígado, aunque puede ser sintetizado por muchos tejidos. La principal reserva de IGF-I es el plasma sanguíneo.

El IGF-I posee dos isoformas, A y B. Estas proteínas han sido secuenciadas en el caballo y guardan homología con otros mamíferos (Nixon y col 1999, Verwilghen y col 2009). Estas moléculas son transportadas por seis

Cuadro 1. Ejemplos de algunas investigaciones sobre terapia regenerativa de tendones y ligamentos en humanos y otras especies animales.

Examples of some investigations on tendon and ligament regenerative therapy in humans and other animal species.

\begin{tabular}{|c|c|c|c|c|c|}
\hline $\begin{array}{l}\text { Especie y tipo de } \\
\text { investigación }\end{array}$ & $\begin{array}{l}\text { Factor de } \\
\text { crecimiento }\end{array}$ & Clase de terapia & Estructura & Resultados & Autor \\
\hline Humano (in vitro) & $\begin{array}{l}\text { TGF- } \beta_{1} \text {, PDGF-A } \\
\text { EGF, bFGF }\end{array}$ & $\begin{array}{l}\text { Diferentes } \\
\text { combinaciones } \\
\text { de GFs }\end{array}$ & $\begin{array}{l}\text { Explantes de } \\
\text { ligamento cruzado } \\
\text { anterior }\end{array}$ & $\begin{array}{l}\text { PDGF-AB } \uparrow \text { proliferación } \\
\text { de fibroblastos } \uparrow \text { Síntesis de } \\
\text { colágeno. bFGF } \uparrow \text { Síntesis de } \\
\text { colágeno }\end{array}$ & $\begin{array}{l}\text { Murray y col } \\
2003\end{array}$ \\
\hline Humano (in vitro) & PDGF & $\begin{array}{l}\text { Combinación con } \\
\text { dexametasona }\end{array}$ & $\begin{array}{l}\text { Explantes tendón } \\
\text { patelar }\end{array}$ & $\begin{array}{l}\text { PDGF contrarresta los efectos } \\
\text { catabólicos producidos por la } \\
\text { dexametasona }\end{array}$ & $\begin{array}{l}\text { Wong y col } \\
2003\end{array}$ \\
\hline $\begin{array}{l}\text { Leporino (in } \\
\text { vivo) Evaluación } \\
\text { biomecánica. }\end{array}$ & TGF- $\beta 1$, PDGF & $\begin{array}{l}\text { Utilización } \\
\text { independiente y } \\
\text { combinada de GFs. }\end{array}$ & $\begin{array}{l}\text { Ligamento colateral } \\
\text { medial de la rodilla }\end{array}$ & $\begin{array}{l}\text { TGF- } \beta 1 \uparrow \text { la fuerza de tensión } \\
\text { de la cicatriz }\end{array}$ & $\begin{array}{l}\text { Spindler y col } \\
2003\end{array}$ \\
\hline Leporino (in vitro) & bFGF & Terapia única & $\begin{array}{l}\text { Explantes de tendón } \\
\text { intrasinovial }\end{array}$ & $\begin{array}{l}\mathrm{bFGF} \uparrow \text { proliferación de } \\
\text { fibroblastos } \uparrow \text { Síntesis de } \\
\text { colágeno }\end{array}$ & $\begin{array}{l}\text { Tang y col } \\
2003\end{array}$ \\
\hline
\end{tabular}

GFs: Factores de crecimiento. TGF- $\beta_{1}$ : Factor de crecimiento transformador beta 1. PDGF: Factor de crecimiento derivado de las plaquetas AB. EGF: Factor de crecimiento epidérmico. bFGF: Factor de crecimiento fibroblástico básico. 
proteínas de unión (IGFPBs) que modulan su acción. El IGF-I se expresa de manera abundante en animales jóvenes, pero su nivel disminuye en caballos viejos (Nixon y col 1999). A nivel articular, el IGF-I induce el aumento de la síntesis de ECM, así como también de colágeno, proteoglicanos y ácido hialurónico (Verwilghen y col 2009) y antagoniza los efectos de la IL-1, por esta razón, estimula la reparación tisular (Platt y col 1998). En seres humanos la compresión articular dinámica que se genera con el ejercicio controlado aumenta las concentraciones de IGF-I en el líquido sinovial y mejora la absorción de este factor de crecimiento en el cartílago (Verwilghen y col 2009). Parece ser que el IGF-I podría actuar de manera similar en tendones y ligamentos equinos (Dahlgren y col 2002, Schnabel y col 2009).

Dahlgren y col (2002) describieron una acción positiva del IGF-I recombinante humano (rh-IGF-I) sobre la reparación tisular de un modelo equino de tendinitis del SDFT inducida con colagenasa. En ese estudio se observó que en las lesiones tratadas con IGF-I se obtenía un mejor registro sonográfico, tenían menor grado de inflamación y se producía una mayor cantidad de colágeno (hidroxiprolina) y ADN que en las lesiones tratadas con solución salina (Dahlgren y col 2002).

El esquema posológico de esa investigación incluyó la aplicación diaria de $2 \mathrm{mg}$ de IGF-I intralesional, durante 10 días (Dahlgren y col 2002). El tratamiento con IGF-I parece esperanzador. Sin embargo, su costo es elevado, por lo que esta terapia podría ser prohibitiva para muchos propietarios. Además, no existe un estudio clínico doble ciego en el que se describa el comportamiento de esta sustancia en caballos con enfermedad natural.

Hormona del crecimiento recombinante equina. La hormona del crecimiento recombinante equina (re-GH) ha sido evaluada en equinos por sus efectos sobre la condición corporal y la recuperación de heridas (Dart y col 2003). Cuando se administra sistémicamente (IM) esta hormona, aumenta la concentración de IGF-I en el plasma. Esto se ve reflejado en el mejoramiento de la condición corporal en yeguas viejas (Dart y col 2003). Los autores de esas investigaciones trabajaron sobre la hipótesis de que la administración exógena de re-GH aumentaría las concentraciones de IGF-I en el líquido sinovial de las articulaciones y modularía el metabolismo del cartílago articular. Los resultados evidenciaron a nivel articular un aumento en las concentraciones de IGF-I. Por tanto, este hallazgo sugeriría una posible aplicación en lesiones de tejidos blandos como tendones y ligamentos en caballos. Sin embargo, en un modelo equino de tendinitis inducido por colagenasa (Dowling y col 2002) se evaluó el efecto de la re-GH. En ese trabajo se demostró que la hormona no alteró el curso de la tendinitis, ni afectó el proceso de reparación, al comparar los miembros tratados con los no tratados.

\section{CONCENTRADO AUTÓLOGO DE PLAQUETAS (PLASMA RICO EN PLAQUETAS)}

Los concentrados autólogos de plaquetas (APC) y el plasma rico en plaquetas son preparados hematológicos que se obtienen por centrifugación de la sangre extraída con anticoagulantes del mismo paciente. Los APCs concentran entre 300 y 600 mil plaquetas/ $\mu$ l (Carmona y col 2011) y el PRP concentra más de un millón de plaquetas/ $\mu$ l (Marx 2004). Ambos (PRP y APC) son una fuente natural de varios GFs, principalmente TGF, TGF-, PDGF, FGF, VEGF y EGF y otras proteínas que modulan la reparación tisular (Anitua y col 2004, Argüelles y col 2006, Carmona y col 2009, Carmona y col 2011).

El PRP se puede obtener por métodos automatizados (aféresis), semiautomatizados o manuales y los APCs, mediante la centrifugación doble en tubo (Carmona y col 2009). En caballos se han documentado por lo menos tres técnicas para obtener PRP. Carter y col (2003) emplearon un método de aféresis, y a partir de un litro de sangre entera con un promedio $120 \times 10^{3}$ plaquetas/ $/$ l obtuvieron una concentración de $490 \times 10^{3}$ plaquetas/ $\mu \mathrm{l}$, y unos niveles de $74,8 \pm 1,31 \mathrm{ng} / \mathrm{ml}$ de TGF- $\beta_{1}$ y $7,45 \pm 0,12 \mathrm{ng} / \mathrm{ml} \mathrm{de}$ PDGF-AB.

Sutter y col (2004) describieron la obtención de PRP equino por medio de dos métodos, un método semiautomatizado y por aféresis (automatizado). La concentración de plaquetas obtenidas con ambas técnicas fue de 855 y 1.472 células/ $\mu 1$, respectivamente. La concentración de IGF-I fue de 183,4 y 107,4 ng/ $\mu \mathrm{l}$, respectivamente. La concentración de TGF- $\beta_{1}$ fue de 23,6 y $15,3 \mathrm{ng} / \mu \mathrm{l}$ y la de TGF- $\beta_{2}$ - fue de 4,3 y $1 \mathrm{ng} / \mu \mathrm{l}$, respectivamente. El número de leucocitos concentrados fue de 33,7 y 32,5 células/ $\mu 1$, respectivamente (Sutter y col 2004).

Se han realizado estudios in vitro (Anitua y col 2005, Smith y col 2006, Schnabel y col 2007, Schnabel y col 2008, Maia y col 2009, McCarrel y Fortier 2009, Bosch y col 2010) que han podido esclarecer el comportamiento $\mathrm{y}$ efecto del PRP/APC en ligamentos y tendones en caballos y seres humanos. Asimismo se han podido realizar descripciones anecdóticas (Sánchez y col 2003) y trabajos clínicos controlados (Abellanet 2009, Carmona y col 2009) de estas sustancias en tendones y ligamentos en las mismas especies (Carmona y col 2011).

El PRP (APC) in vitro incrementa la capacidad proliferativa y metabólica de fibroblastos y tenocitos al aumentar la expresión de colágeno tipo I y la síntesis de proteína oligomérica de matriz del cartílago (COMP) (Smith y col 2006, Schnabel y col 2007, Schnabel y col 2008). En explantes de tendón humano, Anitua y col (2005) demostraron que estas sustancias promovían la angiogénesis mediante el incremento de la expresión del factor de crecimiento vasculoendotelial (VEGF) y el factor de crecimiento hepatocitario (HGF) (Anitua y col 2005). 
En un modelo equino de tendinitis inducida por colagenasa (Maia y col 2009) se observó que a los 36 días los caballos tratados con APCs, obtenidos con un método manual, tenían una mejor disposición histológica de las fibras de colágeno y fibroblastos sobre la matriz de sus tendones en comparación con los caballos del grupo control. Sin embargo, el número de fibroblastos y vasos sanguíneos no fue diferente entre ambos grupos (Maia y col 2009). En otro estudio, Bosch y col (2010) realizaron un estudio sobre la calidad de la reparación de lesiones tendinosas centrales (core lesion) inducidas mecánicamente en caballos tratados con PRP (obtenido mediante un método semiautomatizado). Se observó que a los seis meses el grupo de caballos tratados con APC presentó una mejor arquitectura histológica, mayor contenido de colágeno, glicosaminoglicanos y DNA y mayor resistencia ténsil que el grupo control. Por otro lado, los tendones tratados con PRP estimularon la neovascularización del sitio de la lesión debido a la concentración de factores de crecimiento especialmente VEGF (Bosch y col 2010). En estos dos estudios se puede concluir que tanto el método manual como el semiautomatizado promueven resultados biológicos muy similares (Carmona y col 2011).

Se han realizado descripciones anecdóticas de su uso en patología del tendón de Aquiles en dos atletas y para tratar una avulsión cartilaginosa no traumática en un futbolista (Sánchez y col 2003). Sánchez y col (2007) realizaron un estudio clínico en 12 atletas con lesión del tendón de Aquiles. Un grupo de seis fue sometido a reparación abierta con sutura y PRP, el otro grupo (seis atletas) se trató sin la adición de PRP. Los atletas que recibieron PRP recuperaron su rango de movimiento anterior $7 \pm 2$ semanas frente a $11 \pm 3$ semanas y reanudaron sus actividades en $14 \pm 0,8$ semanas frente a $21 \pm 3$ semanas (Sánchez y col 2007). Los resultados fueron esperanzadores. Sin embargo, es necesario realizar más investigación clínica y molecular en este campo.

De manera hipotética, se podría pensar que la utilización de PRP podría servir para tratar las tendinopatías y desmopatías en el caballo. Se emplearía un tratamiento biológico autólogo con un costo de obtención asequible a la mayoría de los propietarios y se infiltraría una concentración elevada de diferentes GFs en una proporción posiblemente ideal (Dowling y col 2000).

Se ha investigado el efecto clínico de la infiltración intralesional del APC en tendinopatías y desmopatías de caballos (figura 5) (Abellanet 2009, Carmona y col 2009). El método empleado por estos dos autores para obtener los APCs es una técnica de doble centrifugación en la que se puede lograr una concentración de 350-470 $\mathrm{x} 10^{3}$ plaquetas/ $\mu \mathrm{l}$ (Argüelles y col 2006). Carmona y col (2009) encontraron que esta sustancia (APC) mejoraba la apariencia sonográfica y disminuía la cojera asociada con la lesión. Además, los animales retornaron a su nivel de ejercicio normal, más rápido de lo que lo hacen los controles históricos (propios o los descritos en la literatura), tratados con terapia conservadora o con reposo (Carmona y col 2009).

Abellanet (2009) realizó un estudio clínico controlado donde evaluó el uso clínico de APCs (obtenidos mediante el método del tubo (Argüelles y col 2006, Carmona y col 2009) en caballos trotones. 10 caballos con tendinopatía del flexor digital profundo, 16 con lesiones del ligamento suspensorio y 72 con tendinopatía del tendón flexor digital superficial. Los caballos con tendinopatía del tendón flexor digital profundo que recibieron tratamiento con APCs presentaron mejoría del $100 \%$ en comparación con el $0 \%$ del grupo control $(n=4)$ y una recidiva del $17 \%$ en los caballos tratados. Los caballos con desmopatía del ligamento suspensor presentaron una mejoría clínica del $90 \%$ vs $0 \%$ para el grupo control $(n=16)$. De aquellos que fueron tratados con APCs recayó el 10\%. Los caballos con tendinopatía del tendón flexor digital superficial presentaron una mejoría clínica del $80 \%$ vs $45 \%$ para el grupo control $(n=9)$. De los que fueron tratados con APCs recayó el $22 \%$ en comparación con el $80 \%$ del grupo control. Todos los caballos tratados con APCs se recuperaron al cabo de 6 meses (Abellanet 2009).

\section{ASPIRADOS DE MÉDULA ÓSEA Y CÉLULAS MESENQUIMALES}

Herthel (2001) describió la utilización de aspirados de médula ósea como tratamiento de 100 caballos con desmopatía del SL. El 50\% de estos pacientes también fue tratado con fasciotomía plantar. Los resultados obtenidos fueron buenos y más del $85 \%$ de los caballos recuperaron un nivel de función normal a los seis meses y otro 5\% continuó con cojera moderada. Sin embargo, no demostró si la mejoría clínica en esos caballos se pudo presentar por repoblamiento celular o por el efecto modulador de la respuesta biológica, que pudo ser desencadenado por GFs presentes en esos aspirados medulares, o por la fasciotomía que fue realizada en el $50 \%$ de los caballos (Herthel 2001).

El grupo de Smith (Smith y col 2003) fue uno de los primeros en usar células mesenquimales autólogas como tratamiento en lesiones de tendón en caballos. En el 2003 ese equipo (Smith y Schramme 2003) describió una técnica para aislar y replicar células mesenquimales obtenidas de la medula ósea esternal de un caballo de polo con una lesión crónica central grave de un SDFT con una cronicidad de cinco meses de duración. En ese caso, solo se empleó sedación y anestesia local, tanto para obtener el aspirado medular, así como para la posterior aplicación de las células mesenquimales, 16 días posteriores a su obtención. Esos investigadores reexaminaron el paciente a los 10 días y seis semanas posteriores a la infiltración. Las evaluaciones ecográficas mostraron una clara disminución del proceso patológico y una estructura tendinosa casi normal a las seis semanas (Smith y Schramme 2003). 
Los resultados descritos fueron buenos para ese paciente. Sin embargo, no se puede concluir que la implantación de las células mesenquimales haya sido responsable de los cambios ecográficos observados. Es necesario considerar que la calidad de la imagen ecográfica no está correlacionada con la calidad de la ECM del tendón (Dowling y col 2000).

En el 2008 Smith publicó un valioso trabajo donde evaluó el tratamiento con células mesenquimales autólogas en 168 caballos de deporte con lesión en el SDFT. Luego de 48 semanas de rehabilitación, los caballos retornaron a su entrenamiento habitual. De los caballos que se introdujeron a un entrenamiento continuo solo reincidió el 18\% contra un 56\% reportado con terapias convencionales (Smith 2008).

Recientemente, el equipo de Smith (Guest y col 2010) realizó una investigación en ocho caballos con lesión del SDFT, los cuales fueron tratados con células embrionarias alogénicas y con células mesenquimales estromales autólogas. Lo más llamativo de este estudio fue la supervivencia que tuvieron las células embrionarias $(60 \%)$ frente a las células mesenquimales $(<5 \%)$ en la sección inyectada del tendón. Por otra parte, se estableció que no hubo ningún tipo de respuesta inmune en los caballos luego de la aplicación intralesional de las células embrionarias alogénicas (Guest y col 2010).

Crovace y col (2010) realizaron un estudio histológico e inmunohistoquímico en seis caballos, en el que evaluaron el efecto de tres tratamientos: 1) células estromales de medula ósea, 2) células mononucleadas de médula ósea y 3) placebo (solución salina) en un modelo de tendinitis inducida por colagenasa. En los tratamientos 1 y 2 hubo mayor presentación de colágeno tipo I (2,6 y 3,0 unidades semicuantitativas de clasificación histológica (USCH) respectivamente) y menor presentación de colágeno tipo III (1,2 y 1,4 USCH respectivamente). Asimismo, la concentración de COMP se encontró aumentada (2,2 y 2,4 USCH respectivamente). El grupo placebo, por el contrario, experimentó un aumento en la presentación de fibras de colágeno tipo III (2,3 USCH) menor presentación de fibras de colágeno tipo I (1,3 USCH) y menor concentración de COMP (1,3 USCH). Los resultados de este trabajo sugieren una regeneración del tejido lesionado experimentalmente en los tratamientos 1 y 2 , mientras que en el tratamiento 3 sugiere una reparación cicatricial en el área dañada experimentalmente (Crovace y col 2010). Aunque los tratamientos 1 y 2 no difirieron estadísticamente, el posible uso de células mononucleadas derivadas de médula ósea podría ser una opción terapéutica más económica y menos sofisticada para tratar lesiones en tendones equinos con enfermedad natural. Sin embargo, es necesario realizar más estudios donde se obtenga información bioquímica y molecular de estas sustancias y así afianzar su uso en la clínica equina.

\section{CONCLUSIÓN}

Los nuevos conocimientos moleculares del comportamiento fisiopatológico de las lesiones de los tendones y ligamentos del caballo y otras especies animales han permitido desarrollar nuevas estrategias para el desarrollo de tratamientos regenerativos de estas patologías. Sin embargo, solo a través de investigaciones experimentales y clínicas estrictamente planificadas, desarrolladas y evaluadas se podrá conocer el lugar adecuado de cada una de las terapias regenerativas presentadas en esta revisión.

\section{RESUMEN}

Las lesiones de tendones y ligamentos son frecuentes en caballos. Las estructuras más afectadas son el tendón digital flexor superficial (SDFT) y el ligamento suspensor (LS). La forma clínica de estas patologías generalmente es crónica y de naturaleza degenerativa. Los tratamientos comúnmente empleados no producen curación definitiva del problema. Una gran mayoría de pacientes recaen o no vuelven a recuperar su capacidad atlética inicial. El avance del conocimiento molecular de la fisiopatología de las tendinopatías y desmopatías del hombre, caballo y otros animales ha permitido evidenciar la presencia de citocinas catabólicas, las cuales posiblemente sean responsables del trastorno general observado en estas patologías. El objetivo actual del tratamiento de estos problemas es la regeneración y no la reparación (cicatriz) del tejido lesionado. En el caballo se han descrito tratamientos experimentales y clínicos novedosos. Estos tratamientos incluyen la inyección de aspirados de médula ósea, células madre mesenquimales, factores de crecimiento recombinantes como el factor de crecimiento insulínico tipo I y plasma rico en plaquetas (también llamado concentrado autólogo de plaquetas), entre otros. Los resultados observados han sido prometedores cuando estas novedosas terapias han sido empleadas. Sin embargo, al igual que lo que sucede con otros tratamientos, es necesario realizar más investigaciones para demostrar que su uso clínico puede ser efectivo y seguro en caballos.

\section{REFERENCIAS}

Abellanet I. 2009. La terapia de lesiones de tejidos blandos y articulaciones con plasma rico en plaquetas en caballos de deporte: evidencia clínica y bioquímica que valida su utilización. Tesis Doctoral, Facultad de Veterinaria, Universidad Autónoma de Barcelona, España.

Abramowitch SD, M Yagi, E Tsuda, SLY Woo. 2003. The healing medial collateral ligament following a combined anterior cruciate and medial collateral ligament injury-a biomechanical study in a goat model. J Orthop Res 21, 1124-1130.

Anderson TM, CW McIlwraith, P Douay. 2004. The role of conformation in musculoskeletal problems in the racing Thoroughbred. Equine Vet J 36, 571-575.

Anitua E, I Andia, B Ardanza, P Nurden, AT Nurden. 2004. Autologous platelets as a source of proteins for healing and tissue regeneration. Thromb Haemost 91, 4-15.

Anitua E, I Andia, M Sanchez, J Azofra, MM Zalduendo, M de la Fuente, P Nurden, A Nurden. 2005. Autologous preparations rich in growth factors promote proliferation and induce VEGF and HGF production by human tendon cells in culture. J Orthoph Res 23, 281-286.

Arai K, Y Kasashima, A Kobayashi, A Kuwano, T Yoshihara. 2002. TGF- $[\beta]$ alters collagen XII and XIV mRNA levels in cultured equine tenocytes. Matrix Biol 21, 243-250.

Argüelles D, JU Carmona, J Pastor, A Iborra, L Viñals, P Martínez, E Bach, M Prades. 2006. Evaluation of single and double 
centrifugation tube methods for concentrating equine platelets. Res Vet Sci 81, 237-245.

Birch HL, JVB Bailey, AJ Bailey, AE Goodship. 1999. Age-related changes to the molecular and cellular components of equine flexor tendons. Equine Vet J 31, 391-396.

Birch HL, AM Wilson, AE Goodship. 2008. Physical activity: does longterm, high-intensity exercise in horses result in tendon degeneration? J Appl Physiol 105, 1927-1933.

Bosch G, M Moleman, A Barneveld, PR Van Weeren, HTM Van Schie. 2010. The effect of platelet-rich plasma on the neovascularization of surgically created equine superficial digital flexor tendon lesions. Scand J Med Sci Sports, DOI:10.1111/j.1600-0838.2009.01070.x.

Brockes JP, A Kumar. 2008. Comparative aspects of animal regeneration. Annu Rev Cell Dev Biol 24, 525-549.

Carmona JU, M Prades. 2009. Pathophysiology of osteoarthritis. Com Equine 4, 28-40.

Carmona J, M Prades, D Argüelles. 2009. Concentrados autólogos de plaquetas como tratamiento de lesiones de tejidos blandos del aparato locomotor en caballos. Arch Med Vet 41, 77-82.

Carmona JU, C López, CE Giraldo. 2011. Uso de concentrados autólogos de plaquetas como terapia regenerativa de enfermedades crónicas del aparato músculo-esquelético equino. Arch Med Vet 43, 1-10.

Carter CA, DG Jolly, CE Worden, DG Hendren, CJM Kane. 2003. Platelet-rich plasma gel promotes differentiation and regeneration during equine wound healing. Exp Mol Pathol 74, 244-255.

Chang HJ, AE Burke, RM Glass. 2010. Achilles tendinopathy. J Am Vet Med Assoc 303, 188

Crevier-Denoix N, C Collobert, P Pourcelot, J Denoix, M Sanaa, D Geiger, N Bernard, X Ribot, C Bortolussi, B Bousseau. 1997. Mechanical properties of pathological equine superficial digital flexor tendons. Equine Vet J 29, 23-26.

Crovace A, L Lacitignola, R Giacomo, F Edda. 2010. Histological and immunohistochemical evaluation of autologous cultured bone marrow mesenchymal stem cells and bone marrow mononucleated cells in collagenase-induced tendinitis of equine superficial digital flexor tendon. Vet Med Int, doi:10.4061/2010/250978.

Dahlgren LA, AJ Nixon, BD Brower-Toland. 2001. Effects of $\beta$-aminopropionitrile on equine tendon metabolism in vitro and on effects of insulin-like growth factor-I on matrix production by equine tenocytes. Am J Vet Res 62, 1557-1562.

Dahlgren LA, MCH van der Meulen, JEA Bertram, GS Starrak, AJ Nixon. 2002. Insulin-like growth factor-I improves cellular and molecular aspects of healing in a collagenase-induced model of flexor tendinitis. J Orthop Res 20, 910-919.

Dahlgren LA. 2007. Pathobiology of tendon and ligament injuries. Clin Tech Equine Pract 6, 168-173.

Dart AJ, CB Little, CE Hughes, Q Chu, BA Dowling, DR Hodgson, RJ Rose, KA Johnson. 2003. Recombinant equine growth hormone administration: effects on synovial fluid biomarkers and cartilage metabolism in horses. Equine Vet J 35, 302-307.

Denoix J. 1994. Functional anatomy of tendons and ligaments in the distal limbs (manus and pes). Vet Clin North Am Equine Pract 10, 273-322.

Dowling BA, AJ Dart, DR Hodgson, RKW Smith. 2000. Superficial digital flexor tendonitis in the horse. Equine Vet J 32, 369-378.

Dowling BA, AJ Dart, DR Hodgson, RJ Rose, WR Walsh. 2002. The effect of recombinant equine growth hormone on the biomechanical properties of healing superficial digital flexor tendons in horses. Vet Surg 31, 320-324.

Dowling BA, AJ Dart. 2005. Mechanical and functional properties of the equine superficial digital flexor tendon. Vet J 170, 184-192.

Dyson S, R Arthur, S Palmer, D Richardson. 1995. Suspensory ligament desmitis. Vet Clin North Am Equine Pract 11, 177-215.

Dyson S, R Genovese. 2003. The suspensory apparatus. In: Ross M, Dyson S (eds). Diagnosis and management of lameness in the horse. WB Saunders, St Louis, USA, Pp 654-666.
Dyson SJ. 2004. Medical management of superficial digital flexor tendonitis: a comparative study in 219 horses (1992-2000). Equine Vet J 36, 415-419.

Edwards LJ, AE Goodship, HL Birch, JC Patterson-Kane. 2005. Effect of exercise on age-related changes in collagen fibril diameter distributions in the common digital extensor tendons of young horses. Am J Vet Res 66, 564-568.

Gaujoux-Viala C, M Dougados, L Gossec. 2009. Efficacy and safety of steroid injections for shoulder and elbow tendonitis: a meta-analysis of randomised controlled trials. Ann Rheum Dis 68, 1843-1849.

Goodship A, H Birch, A Wilson. 1994. The pathobiology and repair of tendon and ligament injury. Vet Clin North Am Equine Pract $10,323-349$

Guest DJ, MRW Smith, WR Allen. 2010. Equine embryonic stem-like cells and mesenchymal stromal cells have different survival rates and migration patterns following their injection into damaged superficial digital flexor tendon. Equine Vet J 42, 636-642.

Gurtner GC, MJ Callaghan, MT Longaker. 2007. Progress and potential for regenerative medicine. Annu Rev Med 58, 299-312.

Halper J, B Kim, A Khan, J Yoon, P Mueller. 2006. Degenerative suspensory ligament desmitis as a systemic disorder characterized by proteoglycan accumulation. BMC Vet Res 2, 12 .

Henninger R. 1994. Treatment of superficial digital flexor tendinitis. Vet Clin North Am Equine Pract 10, 409-424.

Herthel DJ. 2001. Enhanced suspensory ligament healing in 100 horses by stem cells and other bone marrow components. In: Lameness in the athletic horse. Proceedings of the Annual Convention of the AAEP, Pp 319-321.

Hosaka Y, R Kirisawa, E Yamamoto, H Ueda, H Iwai, K Takehana. 2002. Localization of cytokines in tendinocytes of the superficial digital flexor tendon in the horse. $J$ Vet Med Sci 64, 945-947.

Kaneps AJ. 2007. Surgical options for treating tendon and ligament injuries. Clin Tech Equine Pract 6, 209-216.

Kastelic J, A Galeski, E Baer. 1978. The multicomposite structure of tendon. Connect Tissue Res 6, 11-23.

Kobayashi A, M Sugisaka, K Takehana, M Yamaguchi, EK Iwasa, M Abe. 1999. Morphological and histochemical analysis of a case of superficial digital flexor tendon injury in the horse. J Comp Pathol 120, 403-414.

Maia L, MV de Souza, JI Ribeiro, AC de Oliveira, GES Alves, LD Benjamin, Y Silva, BM Zandim, JDL Moreira. 2009. Plateletrich plasma in the treatment of induced tendinopathy in horses: histologic evaluation. J Equine Vet Sci 29, 618-626.

Marx RE. 2004. Platelet-rich plasma: evidence to support its use. J Oral Maxillofac Surg 62, 489-496.

McCarrel T, L Fortier. 2009. Temporal growth factor release from platelet-rich plasma, trehalose lyophilized platelets, and bone marrow aspirate and their effect on tendon and ligament gene expression. J Orthop Res 27, 1033-1042.

Murray MM, K Rice, RJ Wright, M Spector. 2003. The effect of selected growth factors on human anterior cruciate ligament cell interactions with a three-dimensional collagen-GAG scaffold. $J$ Orthop Res 21, 238-244.

Nam H, Y Park, G Yoon, H Cho, J Lee. 2010. Co-treatment with hepatocyte growth factor and TGF-beta1 enhances migration of $\mathrm{HaCaT}$ cells through NADPH oxidase-dependent ROS generation. Exp Mol Med 42, 270-279.

Nixon A, B Brower-Toland, L Sandell. 1999. Primary nucleotide structure of predominant and alternate splice forms of equine insulinlike growth factor I and their gene expression patterns in tissues. $\mathrm{Am}$ $J$ Vet Res 60, 1234-1241.

Paavola M, P Kannus, TAH Jarvinen, K Khan, L Józsa, M Jarvinen. 2002. Achilles tendinopathy. J Bone Joint Surg Am 84, 2062-2076.

Patterson-Kane JC, DA Parry, HL Birch, AE Goodship, EC Firth. 1997. An age-related study of morphology and cross-link composition of collagen fibrils in the digital flexor tendons of young thoroughbred horses. Connect Tissue Res 36, 253-260. 
Patterson-Kane J, A Wilson, E Firth, D Parry, A Goodship. 1998. Exercise-related alterations in crimp morphology in the central regions of superficial digital flexor tendons from young thoroughbreds: a controlled study. Equine Vet J 30, 61-64.

Pauwels FE, J Schumacher, IG Mayhew, DC van Sickle. 2009. Neurectomy of the deep branch of the lateral plantar nerve can cause neurogenic atrophy of the muscle fibres in the proximal part of the suspensory ligament (M. interosseous III). Equine Vet J41, 508-510.

Peters-Veluthamaningal C, D van der Windt, J Winters, M-dJ B. 2009 Corticosteroid injection for de Quervain's tenosynovitis. Cochrane Database Syst Rev 8, CD005616.

Platt D, JLE Bird, MT Bayliss. 1998. Ageing of equine articular cartilage: structure and composition of aggrecan and decorin. Equine Vet J 30, 43-52.

Pool R, D Meagher. 1990. Pathologic findings and pathogenesis of racetrack injuries. Vet Clin North Am Equine Pract 6, 1-30.

Ross M, S Dyson. 2002. The suspensory apparatus. In: Diagnosis and management of lameness in the horse. Saunders, Philadelphia, USA, Pp 73

Sánchez M, J Azofra, E Anitua, I Andía, S Padilla, J Santisteban, I Mujika. 2003. Plasma rich in growth factors to treat an articular cartilage avulsion: A case report. Med Sci Sports Exerc 35, 16481652 .

Sánchez M, E Anitua, J Azofra, I Andía, S Padilla, I Mujika. 2007. Comparison of surgically repaired Achilles tendon tears using platelet-rich fibrin matrices. Am J Sports Med 35, 245-251.

Schnabel LV, HO Mohammed, BJ Miller, WG McDermott, MS Jacobson, KS Santangelo, LA Fortier. 2007. Platelet rich plasma (PRP) enhances anabolic gene expression patterns in flexor digitorum superficialis tendons. J Orthop Res 25, 230-240.

Schnabel LV, HO Mohammed, MS Jacobson, LA Fortier. 2008. Effects of platelet rich plasma and acellular bone marrow on gene expression patterns and DNA content of equine suspensory ligament explant cultures. Equine Vet J 40, 260-265.

Schnabel LV, ME Lynch, MC van der Meulen, AE Yeager, MA Kornatowski, AJ Nixon. 2009. Mesenchymal stem cells and insulinlike growth factor-I gene-enhanced mesenchymal stem cells improve structural aspects of healing in equine flexor digitorum superficialis tendons. J Orthop Res 27, 1392-1398.

Smith RK, H Birch, J Patterson-Kane, EC Firth, L Williams, W Cherdchutham, WR Weeren, AE Goodship. 1999. Should equine athletes commence training during skeletal development?: changes in tendon matrix associated with development, ageing, function and exercise. Equine Vet J 31, 201-209.

Smith RKW, M Gerard, B Dowling, AJ Dart, HL Birch, AE Goodship. 2002. Correlation of cartilage oligomeric matrix protein (COMP) levels in equine tendon with mechanical properties: a proposed role for COMP in determining function-specific mechanical characteristics of locomotor tendons. Equine Vet J 34, 241-244.

Smith R, M Schramme. 2003. Tendon injury in the horse: current theories and therapies. In Pract 25, 529-539.

Smith RKW, M Korda, GW Blunn, AE Goodship. 2003. Isolation and implantation of autologous equine mesenchymal stem cells from bone marrow into the superficial digital flexor tendon as a potential novel treatment. Equine Vet J 35, 99-102.

Smith JJ, MW Ross, RK Smith. 2006. Anabolic effects of acellular bone marrow, platelet rich plasma, and serum on equine suspensory ligament fibroblasts in vitro. Vet Comp Orthop Traumatol 19, 43-47.

Smith RKW. 2008. Mesenchymal stem cell therapy for equine tendinopathy. Disabil Rehabil 30, 1752-1758.

Souza MV, PR Van Weeren, HTM Van Schie, CHA Van De Lest 2010. Regional differences in biochemical, biomechanical and histomorphological characteristics of the equine suspensory ligament. Equine Vet J 42, 611-620.

Spindler KP, MM Murray, KB Detwiler, JT Tarter, JM Dawson, LB Nanney, JM Davidson. 2003. The biomechanical response to doses of TGF- $\beta 2$ in the healing rabbit medial collateral ligament. J Orthop Res 21, 245-249.

Sutter WW, AJ Kaneps, AL Bertone. 2004. Comparison of hematologic values and transforming growth factor- $\beta$ and insulin-like growth factor concentrations in platelet concentrates obtained by use of buffy coat and apheresis methods from equine blood. Am J Vet Res 65, 924-930.

Tang JB, Y Xu, F Ding, XT Wang. 2003. Tendon healing in vitro: promotion of collagen gene expression by bFGF with NF-kappaB gene activation. J Hand Surg Am 28, 215-220.

Thorpe CT, PD Clegg, HL Birch. 2010. A review of tendon injury: Why is the equine superficial digital flexor tendon most at risk?. Equine Vet $J$ 42, 174-180.

Van Miert ASJPAM. 2002. Present concepts on the inflammatory modulators with special reference to cytokines. Vet Res Commun 26, 111-126.

Verwilghen D, L Vanderheyden, T Franck, V Busoni, E Enzerink, M Gangl, JP Lejeune, G van Galen, S Grulke, D Serteyn. 2009. Variations of plasmatic concentrations of insulin-like growth factor-I in post-pubescent horses affected with developmental osteochondral lesions. Vet Res Commun 33, 701-709.

Waselau M, WW Sutter, RL Genovese, AL Bertone. 2008. Intralesional injection of platelet-rich plasma followed by controlled exercise for treatment of midbody suspensory ligament desmitis in Standardbred racehorses. J Am Vet Med Assoc 232, 1515-1520.

Wong MWN, YYN Tang, SKM Lee, BSC Fu, BP Chan, CKM Chan. 2003. Effect of dexamethasone on cultured human tenocytes and its reversibility by platelet-derived growth factor. J Bone Joint Surg Am 85, 1914-1920.

Woo SLY, AJ Almarza, S Karaoglu, SD Abramowitch. 2008 a Functional tissue engineering of ligament and tendon injuries. In: Atala A, Lanza R, Thomson J, Nerem R (eds). Principles of Regenerative Medicine. Academic Press, San Diego, USA, Pp 1206-1231.

Woo SLY, MB Fisher, AJ Feola. 2008 ${ }^{\mathrm{b}}$. Contribution of biomechanics to management of ligament and tendon injuries. Mol Cell Biomech 5, 49-68.

Zhang F, H Liu, F Stile, M-P Lei, Y Pang, TM Oswald, J Beck, W Dorsett-Martin, WC Lineaweaver. 2003. Effect of vascular endothelial growth factor on rat Achilles tendon healing. Ann Plast Surg 112, 1613-1619. 